\title{
Corela
}

Cognition, représentation, langage

4-2 | 2006

Vol. $4, n^{\circ} 2$

\section{De intermittent du spectacle à intermittent : de la représentation à la nomination d'un objet $\mathrm{du}$ discours}

Julien LONGHI

\section{(2) OpenEdition}

\section{Journals}

Édition électronique

URL : http://journals.openedition.org/corela/457

DOI : $10.4000 /$ corela.457

ISSN : 1638-573X

Éditeur

Cercle linguistique du Centre et de l'Ouest - CerLICO

Référence électronique

Julien LONGHI, « De intermittent du spectacle à intermittent : de la représentation à la nomination d'un objet du discours », Corela [En ligne], 4-2 | 2006, mis en ligne le 15 décembre 2006, consulté le 22 avril 2019. URL : http://journals.openedition.org/corela/457 ; DOI : 10.4000/corela.457

Ce document a été généré automatiquement le 22 avril 2019

\section{(c) (i) (2)(2)}

Corela - cognition, représentation, langage est mis à disposition selon les termes de la licence Creative Commons Attribution - Pas d'Utilisation Commerciale - Partage dans les Mêmes Conditions 4.0 International. 


\title{
De intermittent du spectacle à intermittent : de la représentation à la nomination d'un objet du discours
}

\author{
Julien LONGHI
}

\section{Introduction}

1 Nous voudrions ici analyser le phénomène d'ellipse observé lors de la «crise des intermittents ", par lequel intermittent du spectacle devient intermittent, et ses enjeux sur les processus de nomination, dans un corpus du Monde et du Figaro de mai à octobre 2003. Ces ellipses ne doivent pas être analysées comme la simple marque d'une économie langagière, ni même être résolues par le recours unique au contexte. Certes, dans ce contexte de «crise des intermittents", l'utilisation de intermittent réfèrerait plus logiquement à intermittent du spectacle.

2 Nous rendrons compte de deux dimensions précises qui témoignent de l'originalité de l'ellipse considérée. En premier lieu, une description des dynamiques du sens à l'œuvre dans ces corpus, inspirée de la Théorie des formes sémantiques (TFS) ${ }^{1}$, et mise en relation avec les formations discursives qui en sont à l'origine, montrera que la répartition de ces tournures elliptiques est inégale selon les positionnements énonciatifs. La temporalité du conflit joue également un rôle différent selon ces positionnements. Cela nous amènera à la deuxième dimension de cet article: par une analyse plus précise du cotexte de ces tournures elliptiques (en utilisant en particulier la notion de profilage développée dans la TFS), nous indiquerons les différents processus syntagmatiques qui interagissent avec intermittent et le profilent dans les textes, pour construire une forme sémantique qui tend à devenir non plus la tournure elliptique de la forme de base intermittent du spectacle, mais une forme qui s'autonomise au fil du corpus. Cette mise en évidence intègrera ainsi plus concrètement la textualité du corpus à la dimension plus énonciative et discursive 
repérée en premier lieu, pour mettre en exergue les particularités linguistiques de intermittent. Cette approche sémantico-discursive nous permettra de traiter linguistiquement l'ellipse tout en considérant les enjeux sociolinguistiques qu'elle soulève.

\section{Les dynamiques sémantico-discursives de intermittent}

3 L'analyse morphologique de intermittent fournit des pistes intéressantes pour l'analyse des motivations. Cette motivation, dans le cadre de la TFS, est saisie grâce au concept de motif: avec le motif, "nous entendons donc prendre en compte une certaine couche "morphémique» du sens, dont la portée ne se limite pas aux morphèmes, mais au contraire, et par exemple, se réalise particulièrement bien dès que l'on envisage les mots eux-mêmes, non d'abord comme des types (seraient-ils instables et sujets à déformations réciproques) mais plutôt comme des ouvroirs à motifs $»^{2}$. Intermittent a été emprunté au latin intermittens, participe présent de intermittere. Le verbe est composé du préfixe interet du verbe mittere. Le préfixe inter exprime l'espacement, la répartition, ou une relation réciproque, quant au verbe mittere, il signifie étymologiquement «laisser dans l'intervalle », « interrompre ». Dans la notion d'intermittent l'espacement ou la relation entre deux choses sont dominants. Il est d'ailleurs remarquable que historiquement, en langue, intermittent est un adjectif qui s'applique à quelque chose qui s'arrête et reprend par intervalle, se comportant comme un synonyme de discontinu. Il est ensuite devenu un nom commun (par ellipse du nom travailleur dans travailleur intermittent). L'exemple du dictionnaire Le Robert (1992), intermittent du spectacle, met d'ailleurs en valeur le caractère irrégulier par rapport au spectacle. Dans l'étude du corpus, les énonciateurs font parfois appel à la légitimité génétique et historique de l'objet, en mettant en avant le caractère discontinu du travail des intermittents: intermittent retient donc la dimension « espacement/répartition » de inter en l'appliquant à travail.

Le schéma suivant synthétise les enjeux du sémantisme de intermittent :

Intermittent ( adj.)

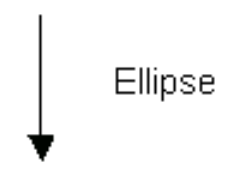

(Travailleur) intermittent (sub.)

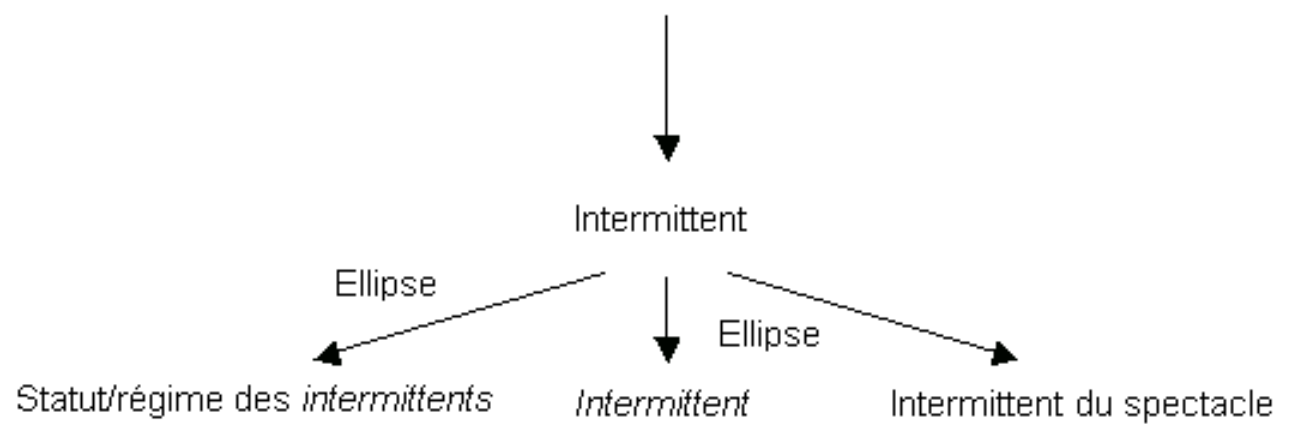


4 Les ellipses qui nous intéressent ici sont celles de la dernière ligne. A ce sujet, nous avons différencié intermittent de régime/statut des intermittents car, comme nous le verrons par la suite, ces structures ne se comportent pas de la même manière en corpus.

\subsection{La construction de la Forme Sémantique intermittent}

5 Lors de l'analyse sémantique de intermittent ${ }^{3}$, nous avons mis en évidence un motif riche, composé de //métier// et //statut//, qui préside aux différentes constructions sémantiques. Ces composantes de motifs, qui sont des fonds, des matériaux ou des supports d'élaboration pour des opérations de profilage et de thématisation, se stabilisent d'une façon plus distincte, plus sélective, par insertion dans des organisations lexicales régionales : à travers la mise en syntagme, et par l'entremise d'opérations textuelles. Pour bien comprendre les implications de cette théorie, il faut prendre en compte son inspiration phénoménologique et gestaltiste, puisque le sens - et la constitution de formes sémantiques - est en relation avec la perception et l'expérience :

Le monde est une constitution compatible avec l'expérience parce qu'il est constitué par l'expérience. Plus particulièrement, la constitution des référents leur est extrinsèque, elle est fondée sur notre expérience. La solution générale de l'articulation sens-référence est alors énonçable avec une extraordinaire simplicité : les objets de la parole sont propres à l'activité linguistique en tant qu'ils sont en partie constitués par la dynamique langagière, mais sont aussi les mêmes que ceux auxquels le langage réfère. Ceci cesse précisément d'être paradoxal dès le moment que le référent n'a d'autre essence que ses propriétés extrinsèques ${ }^{4}$.

6 A la suite des travaux menés en Analyse du Discours en France depuis Pêcheux jusqu'à Foucault, nous considérons en outre que la notion de formation discursive est très importante dès lors que l'on souhaite analyser le sens de formes lexicales construites en discours. Guespin (1976, p. 5) définit par exemple la relation d'appartenance d'un discours à une formation discursive comme facteur constitutif du discours, et ajoute que cette relation est « repérable par l'analyse linguistique ». En reliant l'analyse sémantiques aux positionnements énonciatifs, nous pouvons dégager des grands pôles énonciatifs, qui constituent, dans une étude du sens commun, la modélisation des positions de doxa. Les notions de formation discursive et de formes sémantiques interagissent à notre sens de manière pertinente. En effet,

Une formation discursive, c'est précisément ce qui fait qu'au-delà ou en-deçà du domaine, du genre ou du thème [...] deux discours se ressemblent [...] et que cette ressemblance linguistique témoigne du positionnement idéologico-social de l'instance énonciative (Mayaffre 2004, p. 1)

7 Dans le cadre de l'analyse d'un corpus médiatique, il est intéressant de repérer les ancrages des différents journaux en repérant, pour chaque motivation différente, le comportement de chaque journal. Ceci fait le lien avec la notion de formes sémantiques :

Nous entendons en effet comprendre l'activité de langage sur le mode d'une perception et/ ou d'une construction de formes - de formes sémantiques s'entend [...] Nous cherchons à décrire une dynamique de constitution, de façon telle qu'on puisse la comprendre comme inhérente à l'activité des sujets, tout comme au milieu sémiotique où elle s'exerce (Cadiot et Visetti 2001, p. 48)

8 Combiner une approche discursive à une analyse sémantique permet de relier l'étude du sens aux positions énonciatives qui en sont à l'origine ${ }^{5}$. Pour l'ellipse, cette démarche nous permet de repérer dans quelles rédactions interviennent davantage les tournures 
non-elliptiques ou elliptiques. Ce tableau modélise ainsi les dynamiques sémantiques afférentes aux différentes positions énonciatives ${ }^{6}$ :

\begin{tabular}{|c|c|c|c|}
\hline $\begin{array}{l}\text { POSITIONS } \\
\text { ENONCIATIVES }\end{array}$ & MOTIFS & PROFILS & TOPOI $^{7}$ \\
\hline $\begin{array}{l}\text { Le Figaro } \\
\text { Le Monde }\end{array}$ & //Statut// & $\begin{array}{l}\text { Les intermittents du } \\
\text { spectacle sont privilégiés }\end{array}$ & $\begin{array}{l}\text { Les intermittents sont «fous", } \\
\text { "malades", "insatisfaits", } \\
\text { " pyromanes». }\end{array}$ \\
\hline $\begin{array}{l}\text { Le Monde } \\
\text { Le Figaro }\end{array}$ & $\begin{array}{l}\text { //Statut/ } \\
\text { métier// }\end{array}$ & $\begin{array}{l}\text { Intermittent est un // } \\
\text { statut// qui permet } \\
\text { d'accéder à //métier// }\end{array}$ & $\begin{array}{l}\text { Les intermittents sont "abusés" } \\
\text { et utilisés frauduleusement (ce } \\
\text { sont des permittents). }\end{array}$ \\
\hline Le Monde & $\begin{array}{l}\text { //Métier/ } \\
\text { statut// }\end{array}$ & $\begin{array}{lll}\text { Intermittent } & \text { est un } & / / \\
\text { métier// } & \text { dont } & \text { la } \\
\text { discontinuité } & \text { nécessite } & / / \\
\text { statut// } & & \end{array}$ & $\begin{array}{l}\text { Les intermittents doivent lutter } \\
\text { en permanence (ce sont des } \\
\text { interluttants) }\end{array}$ \\
\hline
\end{tabular}

Comme nous le voyons, les tournures non-elliptiques semblent intervenir davantage dans Le Figaro, où les profilages opèrent sur un motif qui perd sa richesse (//statut// seul ou // métier// seul).

\subsection{Répartition discursive des tournures}

En recherchant grâce au logiciel Lexico ${ }^{8}$ les occurrences de intermittent du spectacle et de intermittent (ainsi que régime des intermittents et statut des intermittents), nous pouvons affiner la tendance repérée précédemment :

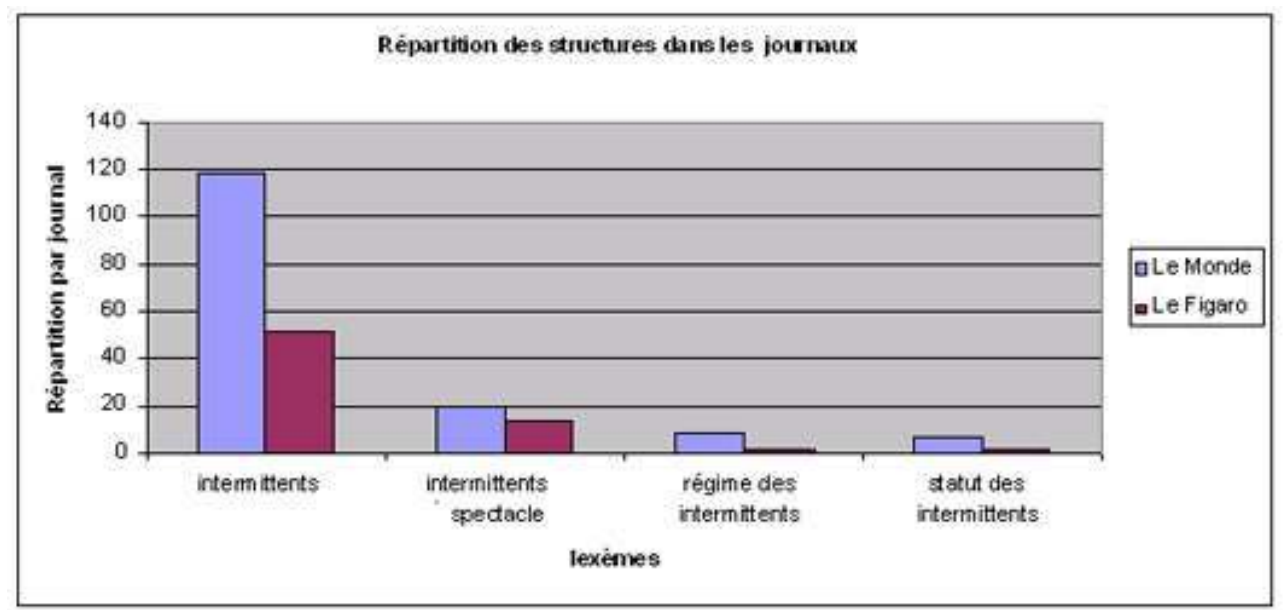

11 Au total, le corpus du Monde compte 3092 occurrences, et celui du Figaro 3467. On trouve 119 occurrences de intermittent dans Le Monde, contre 52 dans Le Figaro. Intermittent du spectacle compte 20 mentions dans Le Monde, contre 14 dans Le Figaro. Ainsi, on relève $16,8 \%$ de tournures non-elliptiques dans Le Monde, et $26,9 \%$ dans Le Figaro. Les tournures elliptiques sont donc proportionnellement plus fréquentes dans Le Monde, où l'on trouve également beaucoup plus de structures « $\mathrm{S}+$ de + intermittent $»(8 / 1$ et $7 / 1$ de régime et 
statut des intermittents). Donc proportionnellement ${ }^{9}$, plus de structures non-elliptiques sont relevées dans Le Figaro, et plus d'ellipses et de « S + de + intermittent » dans Le Monde. Nous interprèterons ces résultats après la mise en valeur de l'influence de la temporalité dans la répartition de ces tournures, afin de pouvoir corréler les différents facteurs.

\subsection{La répartition temporelle des différentes structures}

Pour rendre plus claire la répartition temporelle des tournures, nous avons divisé chaque sous corpus en cinq périodes, qui nous permettent de visualiser l'évolution des formes selon chacune d'elle. Ces périodes ont été déterminées pour chaque corpus selon un critère de répartition homogène du nombre d'articles, afin de pouvoir comparer pour chaque journal l'évolution de la même forme dans une quantité d'article comparable. C'est pourquoi lors de la comparaison que nous ferons des résultats, nous ne pourrons indiquer qu'une différence de tendance dans l'emploi des formes entre les deux journaux, puisque les périodes ne peuvent pas se superposer. Par contre, ce choix méthodologique nous permet, à l'intérieur de chaque journal, de pouvoir analyser l'évolution des formes entre les différentes périodes ${ }^{10}$.

Dans Le Monde, si nous comparons les écarts entre les courbes représentant intermittent et intermittent $d u$ spectacle (afin de savoir le rapport entre ces deux formes), nous remarquons une forte croissance de la tournure elliptique en période 2 (apogée du conflit, avec le durcissement des négociations et les actions multipliées dans les festivals) et également un taux beaucoup plus important en 5 (rentrée culturelle). L'ellipse est donc plus marquée pour les périodes cruciales du conflit. Ces résultats nous invitent à considérer le phénomène elliptique comme lié à des enjeux symboliques, voire idéologiques, dans la mesure où la répartition de formes linguistiques procèdent de conditions de production différentes ${ }^{11}$.

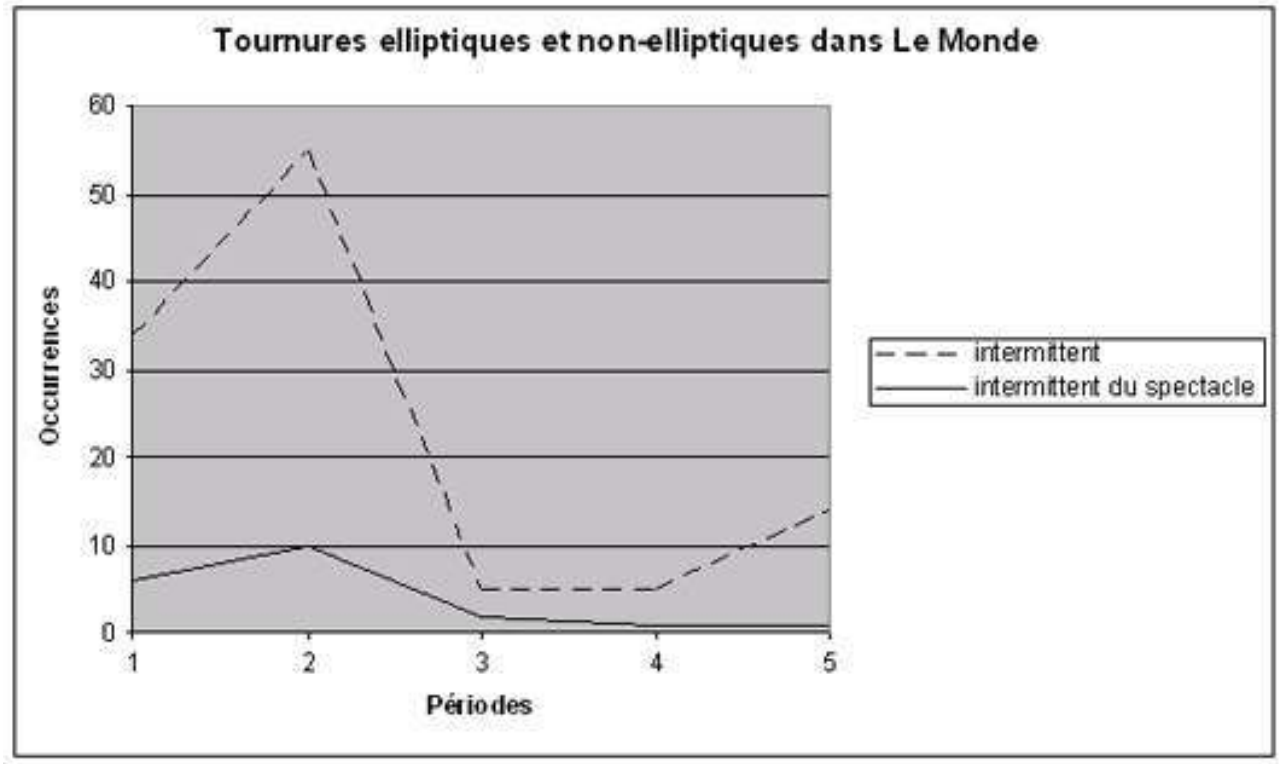

14 Dans Le Figaro, l'écart entre les tournures est croissant des périodes 1 à 4 , diminuant ensuite en période 5. La temporalité semble jouer un rôle dans la mesure où l'on assisterait à un figement de la tournure elliptique. Dans ce cas, intermittent remplacerait intermittent $d u$ spectacle en partie pour des raisons d'économie langagière, le contexte de 
« crise des intermittent » devenant plus établi au fil du temps, de même que la référence faite grâce à intermittent seul.

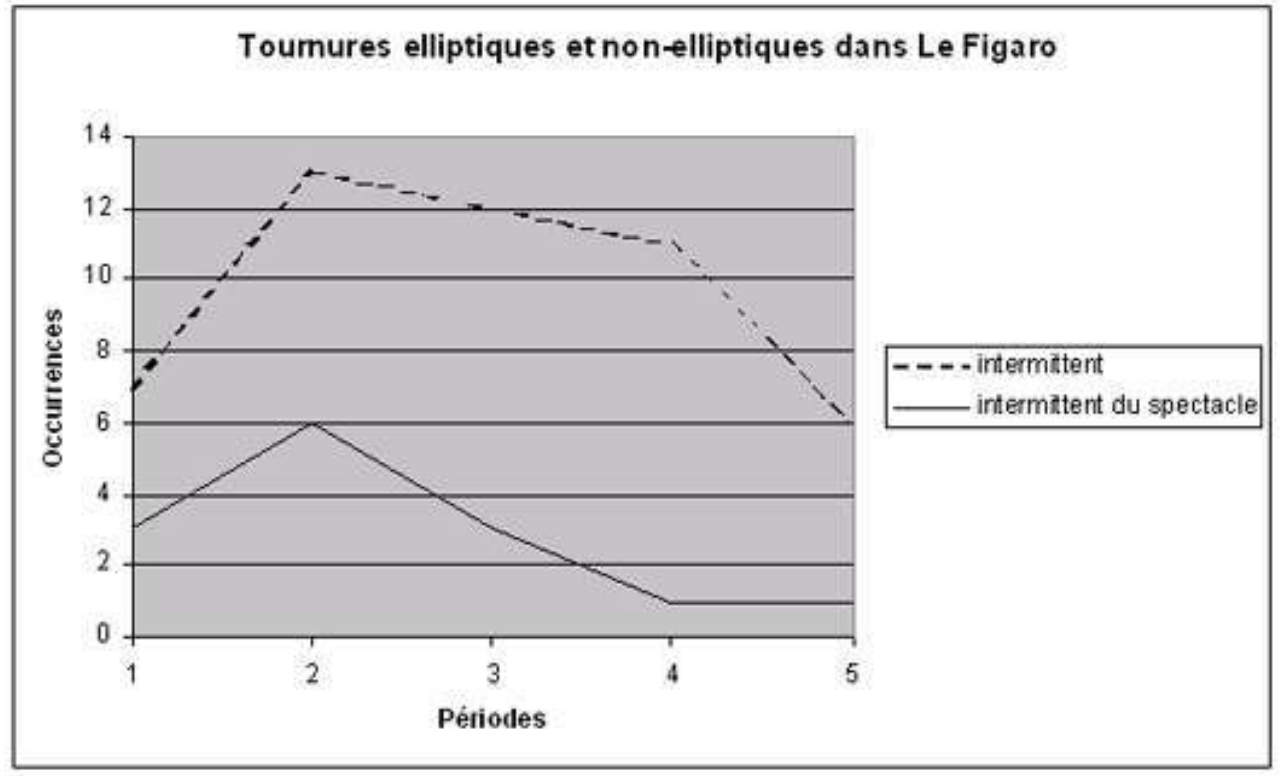

Dans Le Monde, c'est une connivence qui est supposée par l'ellipse : au co-énonciateur de reconstruire ce qui manque, puisque ce manque ne nuit pas à la compréhension. C'est aussi, dans un contexte de conflits sociaux, la création d'un terme emblème, qui tend à se comporter comme le signe d'une lutte sociale. Dans Le Figaro au contraire, l'évolution temporelle indique un figement plus qu'un enjeu idéologique.

\section{Profilages et prise en charge textuelle}

Ces résultats nous invitent à mettre en évidences les procédés syntaxiques et textuels qui permettent d'expliquer ces différences de répartitions des tournures elliptiques et nonelliptiques. Il faut entendre «par profilage, l'ensemble des opérations grammaticales qui contribuent à la stabilisations des unités, et construisent du même coup un ensemble de vues sur la thématique $»^{12}$. Ici, la concurrence avec la structure " $\mathrm{S}+\mathrm{de}+$ intermittent " dans statut des intermittents ou régime des intermittents mérite d'être étudiée : il existe en effet une disproportion étonnante entre les deux journaux (au total 15 structures de ce type dans Le Monde, et seulement 2 dans Le Figaro).

\subsection{Construction « en intension » dans intermittent du spectacle}

Dans intermittent du spectacle, intermittent est inséré dans une structure « $\mathrm{S}+\mathrm{de}+\mathrm{S}$ » intensive : l'objet intermittent est particularisé et spécifié, et la dénomination indique la spécification du spectacle comme constitutive. Le Figaro traite donc davantage du cas plus particulier des intermittents du spectacle, et s'en tient à ceux-ci pour traiter l'information. L'évolution temporelle de l'apparition des tournures corrèle cette hypothèse, puisque la régularité de la croissance des tournures elliptiques indique que intermittent subit davantage l'effet d'un figement (au cours du conflit l'ellipse serait de plus en plus naturelle) que d'influences idéologiques. 


\subsection{Construction « en extension » dans statut/régime des intermittents} au cours de l'évolution du conflit), une forme sémantique autonome, c'est-à-dire une forme qui ne serait plus simplement la tournure elliptique de intermittent du spectacle. Nous avancerons trois argument pour étayer cette thèse : deux concernent l'apparition des néologismes interluttant et permittent ${ }^{13}$; le troisième implique l'évacuation de la composante /métier/ du motif, qui coupe intermittent de son lien avec le spectacle.

\subsection{Le néologisme interluttant}

Le néologisme interluttant conduit à la création d'un topos les intermittents doivent toujours être en lutte

Exemple :

La coordination avignonnaise des interluttants a tenu une assemblée générale (Le Monde 6-7 juillet) 

élément de langue, à la fois du côté morphologique, mais également en rapport avec le travail des topoï. Au niveau morphologique, l'objet conserve le morphème inter, qui se combine avec une composante phonétique en -ant (le jeu de mot utilise en effet la sonorité des lexèmes intermittent et interluttant, bien que l'orthographe diffère d'un terme à l'autre). Le motif nous renseigne sur la motivation sémantique à l'œuvre; les profilages le stabilisent, et le plongent en discours où ils cristallisent la lutte inhérente au motif // métier/statut//, puisque lutter est une nécessité pour ne pas le dégrader. Cette lutte devient intrinsèque à l'objet, modifiant le processus de nomination. Ce néologisme se fonde sur le lexème intermittent, et ne retient donc pas la composante du spectacle, absente de la tournure elliptique. Ainsi, ce néologisme témoigne de l'autonomie prise par intermittent, qui devient la base pour la création d'un élément de langue. En outre, le sémantisme de interluttant ne retient pas non plus la composante du spectacle, et confirme ce que nous disions sur les enjeux idéologiques liés à intermittent (comme élément emblématique dans des discours de conflits sociaux).

\subsection{Le néologisme permittent}

Certains intermittents ou employeurs considèrent prioritairement la composante // statut// du motif: c'est elle qui conditionnerait //métier//, et qui conduirait donc à la fraude et à l'utilisation illégale du système : le motif est alors //statut/métier//.

Exemples

[...] recours de façon abusive aux « permittents » - autrement dit des intermittents permanents (Le Monde, $1^{\text {er }}$ juillet)

Nous devons faire face à la pression de faux intermittents (Le Figaro, 18 juillet, une comédienne)

La création du néologisme (mot-valise) permittent cristallise ce point de vue sur le motif, et rend l'objet paradoxal (car il résulte de intermittent et permanent, qui s'opposent). C'est pour cela que cette motivation est celle de "faux intermittents ». Ici non plus, du spectacle n'est pas intervenu dans l'apparition de ce mot valise, et le sémantisme de permittent ne retient pas cette composante.

\subsection{L'évacuation de la composante //métier//}

Chez certains énonciateurs, la composante //métier// du motif est évacuée. Intermittent se trouve composé de //statut//: c'est un statut qui n'est pas directement lié à la fréquence de travail. C'est cette déviation de point de vue qui permet de poser la question de la légitimité de l'intermittence. Cela peut aller, pour le motif, jusqu'à une réduction du motif en //statut// seul.

Exemple

Les élèves de l'école du Théâtre national de Bretagne, «de futurs ex-intermittents » (

Le Monde, 9 juillet)

Les futurs comédiens seront, selon ce point de vue, dépourvus du statut actuel, qui semble constituer l'essence de l'objet. L'appartenance au monde du spectacle est totalement évacuée, et l'ellipse témoigne de l'évolution sémantique de intermittent, devenu une forme sémantique autonome. 


\section{Conclusion} intermittent $d u$ spectacle, que ce phénomène linguistique recouvre des dimensions qui n'étaient pas perceptibles a priori. En effet, la répartition temporelle et discursive des différentes tournures indique que l'utilisation de l'une ou l'autre des tournures n'est pas indifférente. Le Monde fournit plus de constructions «en extension» de intermittent, indiquant une connivence plus marquée avec le lectorat, et une importance idéologique du choix opéré. Dans Le Figaro, les tournures «en intension » sont plus présentes: les discours concernent davantage l'objet particulier, et la temporalité est plus importante que dans Le Monde.

Enfin, l'activité de nomination va ici jusqu'à l'apparition de néologismes et de formes sémantiques, qui attestent que intermittent tend à se comporter comme une forme sémantique autonome, et non plus comme la simple tournure elliptique de intermittent $d u$ spectacle. La créativité plus générale de l'ellipse est ainsi apparue à travers l'exemple de intermittent : bien plus qu'une simple place vide, l'ellipse recouvre des enjeux discursifs et sociolinguistiques, qui s'intègrent dans l'activité langagière en générale, et qui sont à mettre en rapport avec les apports des autres plans linguistiques.

\section{BIBLIOGRAPHIE}

Bourdieu P., 2001. Langage et pouvoir symbolique, Le Seuil, coll. « Points Essais »

Cadiot P. et Lebas F., 2003. « La constitution extrinsèque du référent » et « Monter et la constitution extrinsèque du référent ", Langages, 150 : 3-30.

Cadiot P. et Visetti Y.-M., 2001. Pour une théorie des formes sémantiques, PUF, coll. « Formes sémiotiques »

Cadiot P. et Visetti Y.-M., 2001-2. « Motifs, profils, thèmes : une approche globale de la polysémie », Cahiers de lexicologie, $79:$ 5-46

Guespin L., 1976. « Type de discours ou fonctionnements discursifs ? ", Langages, 4 :3-9

Longhi J., 2006a. « Permittent et interluttant, deux néologismes entre lexique et discours », Cahiers du L.C.P.E., 7 : 95-109

Longhi J., 2006b. « Le discours dans l'analyse de corpus : doxa et évolution des topoï », Actes du colloque « Col'doc 05 », Université de Paris X, à paraître

Mayaffre D, 2004. « Formation(s) discursive(s) et discours politique : l'exemplarité des discours communistes versus bourgeois durant l'entre-deux-guerres ", Texto !. Accessible en ligne à l'URL : http://www.revue-texto.net/Inedits/Mayaffre/Mayaffre_Formations.html (consulté le 20/05/05) Sarfati G.-E., 1996. « La sémantique : de l'énonciation au sens commun. Éléments d'une pragmatique topique », Texto ! Accessible en ligne à l'URL : http://www.revue-texto.net/Inedits/ Sarfati/Sarfati_Semantique.html (Consultée le 10/01/05)

Corela, 4-2 | 2006 


\section{NOTES}

1. Cadiot et Visetti (2001). Dans la TFS, les objets du langage sont considérés comme des formes stabilisées à la suite de parcours dynamiques, une forme sémantique pouvant être saisie lors de certaines phases, appelées motifs, profils, et thèmes. Ils définissent «les motifs linguistiques comme des germes de signification chaotiques et/ou instables, et le profilage comme un système, déjà frayé et enregistré en lexique et en grammaire, de parcours de stabilisations différentielles pour des lexèmes en interdéfinition" (2001-2, p. 6). La thématique, à prendre dans un sens textuel, renvoie à "ce dont on parle ", à l'ensemble de ce qui est "posé " par l'activité de langage. Dans notre perspective discursive, le concept de thème est remplacé par celui de topos, plus adéquat selon nous pour saisir les spécificités idéologiques et argumentatives du sens. Nous développerons plus longuement ces concepts quand cela sera nécessaire.

2. Cadiot et Visetti (2001, p. 114). Par exemple, pour arbre, les motifs seraient //branchement/ ramification// et //force/stabilité//.

3. Voir à ce sujet Longhi 2006a, pour le traitement sémantique détaillé de intermittent : //métier/ statut// serait le motif historique et génétique : intermittent désigne ainsi un //métier// qui s'accompagne d'un //statut// spécifique. La remise en cause de ce motif peut venir du sens de travail: la convocation d'un topos «le travail enrichit» contredit la nécessité de //statut//, puisque dans ce cadre //métier// devrait suffire, et être l'unique motivation de intermittent. La composante //statut// peut également être niée, par l'emploi de intermittent comme synonyme d'artiste, considérant alors que //métier// (lié à l'art), doit suffire à le motiver. La composante // statut// devient un non-sens, et le motif se compose uniquement de //métier//.Une autre attitude est de considérer prioritairement la composante //statut// du motif: c'est elle qui conditionnerait //métier//, et qui conduirait donc à la fraude et à l'utilisation illégale du système : on a alors //statut/métier//. Chez d'autres énonciateurs, la composante //métier// est évacuée du motif, qui se trouve dès lors composé de //statut//, comme nous le verrons en 3.3.

4. Cadiot et Lebas (2003, p. 5)

5. Dans Longhi 2006b, nous montrons plus en détail l'importance du Discours dans l'analyse de corpus, plus particulièrement pour le lexème libéralisme dans des discours politiques.

6. Les différentes tailles de polices pour les noms de journaux indiquent les différences de proportions des motifs pour chaque rédaction.

7. Sans nous attarder sur la terminologie propre à la sémantique du sens commun développée par Sarfati, nous retiendrons la définition suivante: "on posera que la différence de statut théorique, entre sens commun et topos, dans le cadre des orientations actuelles de la sémantique de l'énonciation, tient précisément à la place qu'ils occupent tous deux dans le nouveau modèle : tandis que le sens commun est le concept organisateur du paradigme topique, celui de topos serait son concept général descriptif - ses toutes premières occurrences ayant marqué, dès leur apparition, le dépassement du paradigme pragmatique au profit d'une perspective encore inédite. Enfin, on réservera le concept de doxa à la délimitation d'une région du sens commun, comme telle dotée de son dispositif de topoï spécifiques » (Sarfati, 1996, III, p. 5).

8. Nous faisons un usage très simple de ce logiciel : il nous permet simplement de comptabiliser le nombre d'occurrences des formes recherchées par corpus, puis par période.

9. Par proportionnellement, nous indiquons que le nombre de formes non elliptiques par rapport à celui de formes elliptiques est très différent dans les deux corpus, puisque sur le total des occurrences de intermittent $16,8 \%$ sont non elliptiques dans Le Monde, contre $26,9 \%$ dans Le Figaro , soit $62 \%$ de plus (alors que si l'on ne regardait que les nombres d'occurrences, on trouverait évidemment plus de formes non elliptiques dans Le Monde, puisqu'il fournit beaucoup plus d'occurrences au total) 
10. Pour Le Monde : $1=13$ juin à $1 \mathrm{er}$ juillet ; $2=2$ à 12 juillet ; $3=15$ juillet à début août ; $4=$ août ; $5=$ septembre ; pour Le Figaro: $1=30$ juin à 1er juillet; $2=2$ à 9 juillet ; $3=9$ à 15 juillet ; $4=$ jusqu'à fin juillet; $5=$ jusqu'à fin août. Ces différences nous montrent en outre l'attention différente portée par ces journaux à un même phénomène, la rentrée culturelle faisant par exemple l'objet d'un traitement conséquent dans Le Monde qui n'a pas trouvé d'équivalent dans Le Figaro.

11. Pour Guespin (1976, p. 4-5), « un regard jeté sur un texte d'un point de vue de sa structuration « en langue » en fait un énoncé ; une étude linguistique des conditions de production de ce texte en fera un discours" : ici l'évolution du conflit modifie les conditions de productions de chaque article, et s'accompagne d'une évolution des formes linguistiques, ce qui nous permet de poser une relation entre les conditions de productions et les tournures elliptiques/non elliptiques.

12. Cadiot et Visetti (2001, p. 127)

13. D'une manière fréquentielle, ces néologismes sont assez marginaux, puisque qu'une seule occurrence de permittent est recensée dans Le Monde ; interluttant est plus fréquent, puisqu'il est utilisé quatre fois dans Le Monde et deux fois dans Le Figaro. Cependant, leur simple existence, et surtout l'usage qui semble en être fait -selon les journaux - dans la communauté culturelle, nous incite à les considérer avec attention.

\section{RÉSUMÉS}

Nous analysons ici le phénomène d'ellipse observé lors de la "crise des intermittents ", dans un corpus du Monde et du Figaro de mai à octobre 2003 : lors de cette très courte diachronie, un phénomène d'ellipse est apparu, par lequel intermittent du spectacle devient intermittent. Nous rendons compte de deux dimensions précises qui témoignent de l'originalité de l'ellipse considérée. Une description des dynamiques du sens à l'œuvre dans ces corpus, en relation avec les formations discursives qui en sont à l'origine, montrera que la répartition de ces tournures elliptiques est inégale selon les positionnements énonciatifs. La temporalité du conflit joue également un rôle différent selon ces positionnements. Cela nous conduit à une analyse plus précise du cotexte de ces tournures elliptiques: nous indiquons les différents processus syntagmatiques qui interagissent avec intermittent et le profilent dans les textes.

We analyze the phenomenon of ellipsis observed during the french "crisis of intermittent", in a corpus of Le Monde and Le Figaro from May to October 2003: during this short diachrony, a phenomenon of ellipsis appeared: intermittent du spectacle becomes intermittent. We show two dimensions which testify to the originality of this ellipsis. A description of the dynamics of the meaning in these corpora, in relation with the discursive formations which are in the beginning, will show that the distribution of these elliptic structures depends on the enunciative positions. The temporality of the conflict is also important. That invites us to a more precise analysis of the cotexte of these elliptic structures: we indicate the various syntagmatic processes which interact with intermittent and profile it in the texts. 
INDEX

Mots-clés : nomination, formation discursive, ellipse, néologisme, forme sémantique

Keywords : nomination, discursive formation, ellipsis, neologism, semantic form 